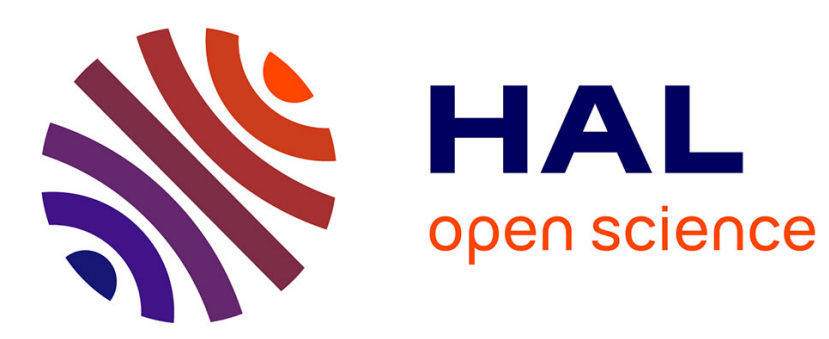

\title{
Vibration control of the beam of the future linear collider
}

\author{
B. Caron, G. Balik, L. Brunetti, A. Jérémie
}

\section{To cite this version:}

B. Caron, G. Balik, L. Brunetti, A. Jérémie. Vibration control of the beam of the future linear collider. Control Engineering Practice, 2012, 20, pp.236-247. 10.1016/j.conengprac.2011.11.001. in2p3-00638748

\author{
HAL Id: in2p3-00638748 \\ https://hal.in2p3.fr/in2p3-00638748
}

Submitted on 7 Nov 2011

HAL is a multi-disciplinary open access archive for the deposit and dissemination of scientific research documents, whether they are published or not. The documents may come from teaching and research institutions in France or abroad, or from public or private research centers.
L'archive ouverte pluridisciplinaire HAL, est destinée au dépôt et à la diffusion de documents scientifiques de niveau recherche, publiés ou non, émanant des établissements d'enseignement et de recherche français ou étrangers, des laboratoires publics ou privés. 


\title{
Vibration control of the beam of the future linear collider
}

\author{
B.Caron $^{1 *}$, G. Balik ${ }^{2}$, L.Brunetti ${ }^{2}$, Andrea Jeremie ${ }^{2}$ \\ 1: SYMME-Polytech'Annecy Chambéry - Université de Savoie - Annecy-le-Vieux France \\ 2: LAPP-IN2P3-CNRS - Université de Savoie - Annecy-le-Vieux France \\ *. bernard.caron@univ-savoie.fr
}

2 May 2011

\begin{abstract}
ARTICLE INFO
ABSTRACT

Article history: This paper proposes a new approach for beam stabilization of the future Compact LInear

Received Collider (CLIC). The method attempts to increase the efficiency of traditional methods. It is

Accepted

Available online

Keywords:

Adaptive algorithm

Disturbance rejection composed of a hybrid adaptive filtering algorithm that uses both feedback and adaptive Least-squares algorithm control. The scheme uses an estimate of the prediction error to update the adaptive filter's Optical feedback parameters. The strategy of this method is described considering the process environment.

Parameter optimization

Noise control

Vibration measurement The method efficiency is evaluated, and it is demonstrated that it provides high damping, fast vibration suppression, good robustness and easy realization thanks to the simplicity of the computations.
\end{abstract}

\section{Introduction}

The search for the infinitely small requires large instruments, as is the case in particle physics. One of the projects currently being studied is the construction of the Compact LInear Collider (CLIC) (Braun, 2008), composed of two faceto-face, $21 \mathrm{~km}$-long arms. An electron beam and a positron beam are accelerated respectively in the two arms with transverse spot sizes in the nanometer range, before colliding at the center of the machine. The quantity commonly used to characterize an accelerator is the luminosity [proportional to the number of particles per unit area per unit time ]. It links the interaction rate to the interaction cross section (luminosity is proportional to the number of particle in a bunch and inversely proportional to the cross section of the bunch). The discovery potential depends on the value of the obtained luminosity, which strongly depends on the relative beam-beam offset (Dalena, 2010). Transporting the CLIC beams over $21 \mathrm{~km}$, focusing them to nanometer spot sizes and colliding the two distinct beams at the collision interaction point with a 
horizontal crossing angle of $20 \mathrm{mrad}$ (Schulte, 2001) imposes extraordinary requirements on the stability of the magnetic fields.

This study aims at positioning the beams to the sub-nanometer level after passing through the last two focusing magnets, called Final Focus magnets (FF). As the shape of the beam is elliptic, its vertical dimension being smaller than its horizontal one, requirements on the vertical position of the beam are tighter. Therefore, the most critical case is studied. Specifications require the vertical offset between the beams to be lower than $0.1 \mathrm{~nm}$ (integrated RMS, see part 2.2). Different methods are usually carried out: one consists in controlling the vertical displacement of the various mechanical elements in order to minimize motion, another one consists in controlling the beam trajectory by using magnetic fields.

The first part of the paper aims at describing the process, its environment, and particularly the disturbances which will impact the beam motion and the classical methods used on the other colliders. The second part of this paper is a description of the beam-based feedback, using a linear controller. The last part describes the proposed adaptive control feedback combined with a mechanical filter in order to lower the disturbances.

\section{Description of the future Compact LInear Collider}

\subsection{Description of the process}

The future linear collider project CLIC will be composed of two linear accelerators of about 21 kilometers long that produce particle trains with the required energy and low emittance (Assmann, 2000a). CLIC accelerates the beams through the longest part of the accelerator called the Main Linac (ML) to the desired energy, then it focuses the beams to a vertical spot size down to a few nanometer and steers them into collision at the Interaction Point (IP), where the particle detector is placed, in order to obtain the target luminosity. This scheme is detailed in figure 1.

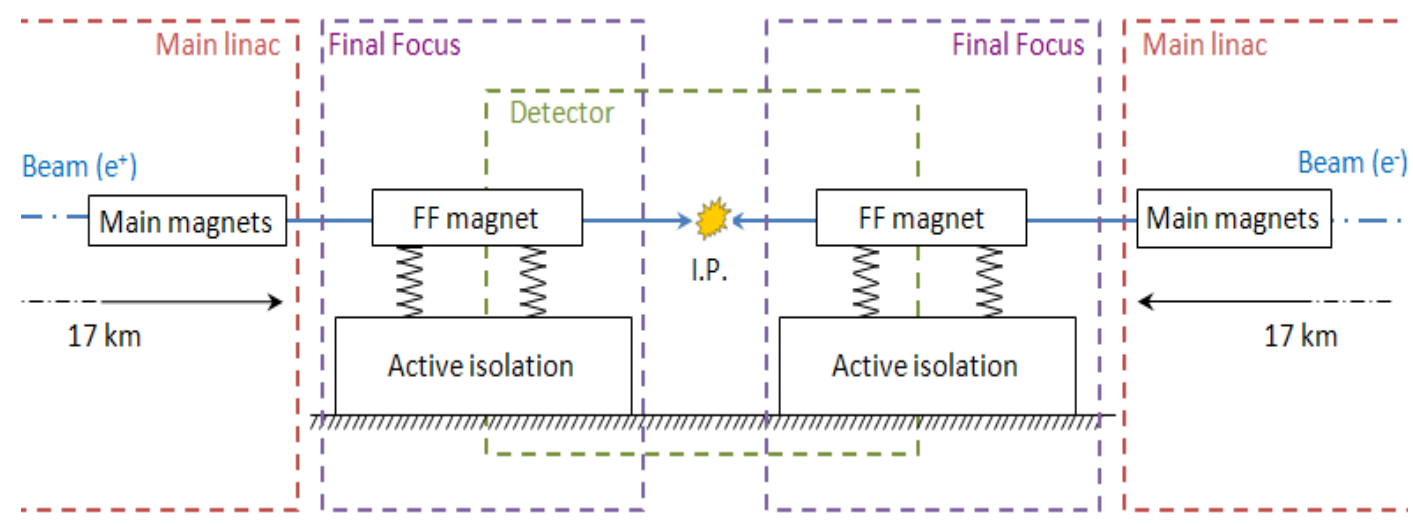

Fig. 1: Scheme of the future compact linear collider (CLIC) at the interaction point 
The final focusing down to the nanometer scale of each beam is done by the last FF magnets. The beam is composed of series of beam trains separated from each other in time by 20 ms. Figure 2 represents the beam structure.

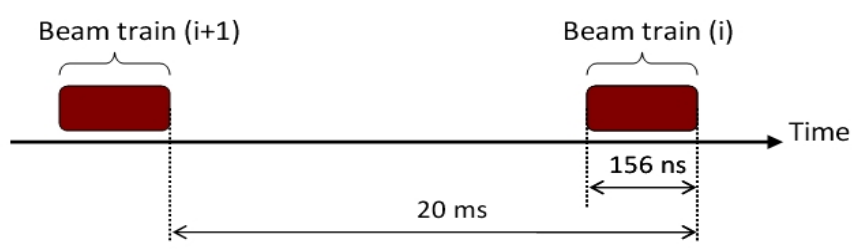

Fig. 2: Beam structure

This study aims at developing a method which ensures the reduction of the vertical beam displacement at the interaction point, in order to meet the requirements. Given the beam size, colliding two beams at the interaction point is a very complex challenge because achieving this goal depends on a lot of parameters. On the one hand, considering that the beams have to be located face to face (in the horizontal plane) during the collision, the position of the focused magnets has to be strictly at the same height. It results in a tight alignment of all elements at a micrometer scale using different possible methods, dedicated to the alignment of the various magnets (Sjöström, 2007), and the detector (Goldstein, 2003). On the other hand, the motion of the beam has to be lower than a tenth of nanometer, thus, different methods are carried out in order to reduce as much as possible the impact on the beam of the disturbance source.

\subsection{Ground motion}

Ground motion is the main disturbance of the beam-line because it is directly transmitted from the ground to the magnets via their support. However, its influence has been studied extensively (Adloff, 2005). Two phenomena can be considered. On the one hand, there is the Earth motion, for frequencies lower than $1 \mathrm{~Hz}$, due to swell, tectonic motion, atmospheric changes, and on the other hand, there is cultural noise (for frequencies above $1 \mathrm{~Hz}$ ) due to human activities which can drastically change from site to site. The PSD of the natural ground motion is a steep function of frequency $f$ which falls off as $1 / f^{4}$. Several peaks can be observed, and are related to machinery and structural resonances. The PSD is particularly useful, because it can help in calculating the total RMS (Root Mean Square) displacement $Z_{r m s}$ in any frequency band (or indeed over the whole frequency range measured) by integrating the displacement PSD $\left(S_{x}\right)$ and taking the square root :

$$
\mathrm{Z}_{\mathrm{rms}}(f)=\sqrt{\int_{f_{0}}^{f_{\max }} S_{x}(f) d f} .
$$

The integrated RMS gives the sum of the RMS values observed for all frequencies between $f_{0}$ and $f_{\max }$. 
In figure 3, the curves represent the PSD and integrated RMS displacement of the ground motion obtained at LAPP and at the Compact Muon Solenoid (CMS) (CMS, 2008) experimental hall which is one of the multi-purpose detectors on the Large Hadron Collider (LHC) (Virdee, 2010).

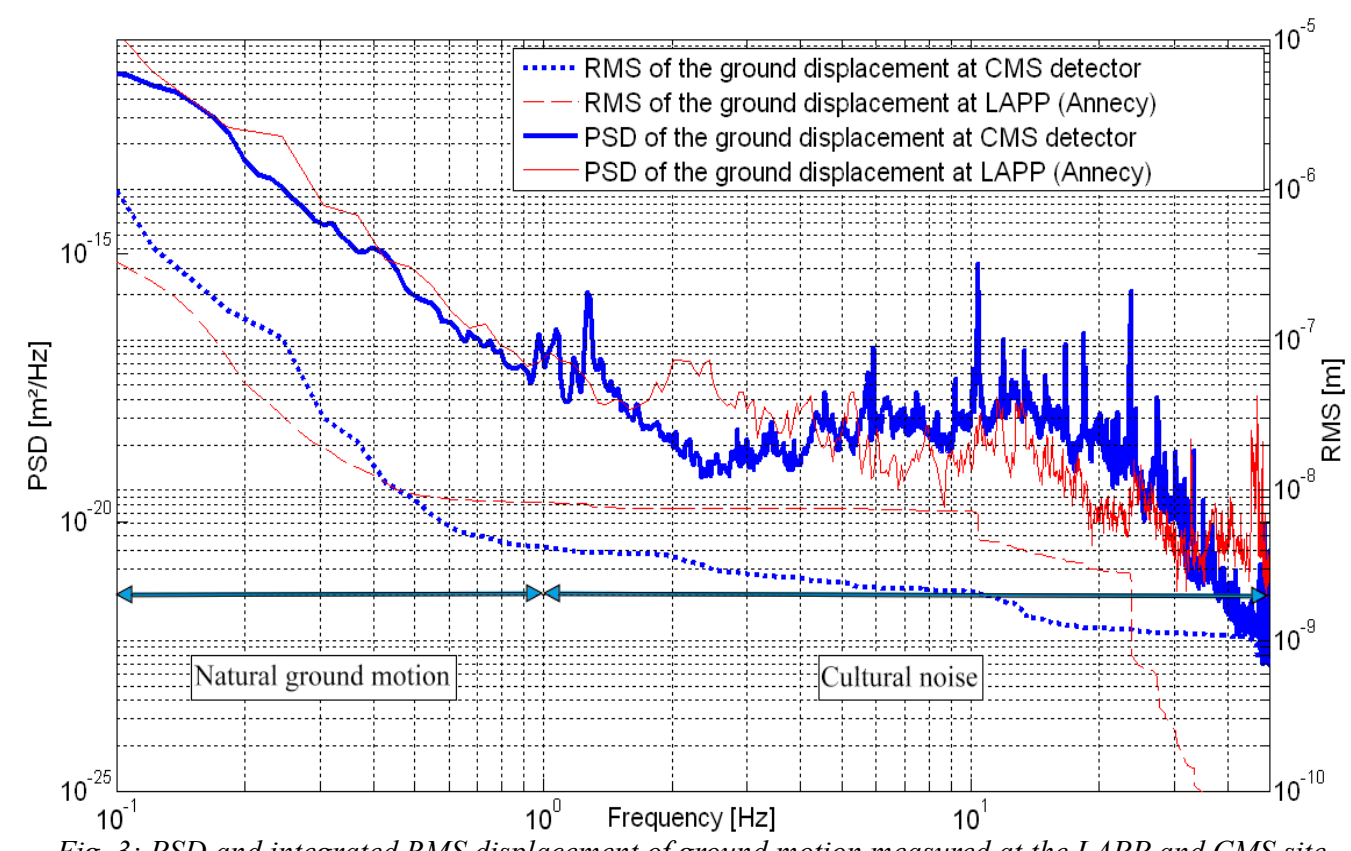

Fig. 3: PSD and integrated RMS displacement of ground motion measured at the LAPP and CMS site

The ground motion depends on the location in the world (Bialowons, 2006). Geophones (Güralp ${ }^{1}$ CMG-6T calibration: $\pm 12.5 \mathrm{~mm} . \mathrm{s}^{-1}$, frequency range: [0.033-50] Hz, sensitivity: 2x998 V.s.m- ${ }^{1}$, resolution: 0.05 nanometer between $4 \mathrm{~Hz}$ and $50 \mathrm{~Hz}$ ) were used to measure the CMS ground motion. Given the limited bandwidth of the geophones, simulations have been performed in the frequency range $[0.1-50] \mathrm{Hz}$ instead of $[0-\infty[$. Note that the $50 \mathrm{~Hz}$ limitation has no impact on the results as the PSD decreases significantly with frequencies above $50 \mathrm{~Hz}$. Sensor signals including amplification were recorded by the PULSE² acquisition system. This set-up guarantees a good resolution in the selected bandwidth (Bolzon, 2005), a very low noise of the measurement chain (Bolzon, 2008), and ensures very accurate measurements of ground motion even for a quiet site. Temporal data have been implemented in Matlab/Simulink ${ }^{3}$ to simulate the effect of ground motion on the plant.

\subsection{Direct disturbances on the FF magnets}

Two main disturbances other than ground motion have a direct effect on the FF magnets: the acoustic pressure and other processes (such as the cooling system (Redaelli, 2002)) linked to the FF magnets. Since all components are not known, 1 Güralp Systems Limited, Inc.

2 Brüel \& Kjaer Company, controller 7537A.

The MathWorks, Inc. 
the acoustic pressure has been measured in a laboratory environment and its effects can be modeled by a pink noise on a large bandwidth (Bolzon, 2006). Internal disturbances due to surrounding processes (cooling system, vacuum pumps) usually cause a harmonic motion at certain frequencies. The impact of these disturbances on the beam displacement depends on the magnet structure, which is being developed. Moreover, the CLIC intensity cannot be quantified without more information concerning the magnets' location or their environment. As the beam motion is most likely due to the ground motion disturbance, other disturbance sources will be neglected. A robustness study is described later in this paper.

\subsection{The studied FF system}

The FF system is the most downstream part of a linear collider, which focuses the beam to a very small spot size. At this point, the beam goes through a final focusing magnet subject to disturbances. This magnet stands on an active/passive support designed to reduce ground motion vibrations. The global structure is summarized in figure 4 .

Elements of this process (sensors, actuators, magnets) are currently being developed. The concept uses a post-collision Beam Position Monitor (BPM) (Burrows, 2007) downstream from the IP to obtain the beam-beam offset at the IP by inferring the deflection angle (Bambade, 1989). The BPM consists essentially in four electrodes attached to either side of the vacuum tube in which the beam circulates. An electron beam passing through a BPM causes a charge on the electrodes, which depends on the beam position. Since it is located at a distance of $3.5 \mathrm{~m}$ from the IP, the post-collision BPM measures a highly amplified image of the relative displacement or beam-beam offset $\Delta Y$. Thus, it is assumed that the ground motion vibration disturbances have a negligible impact on it.

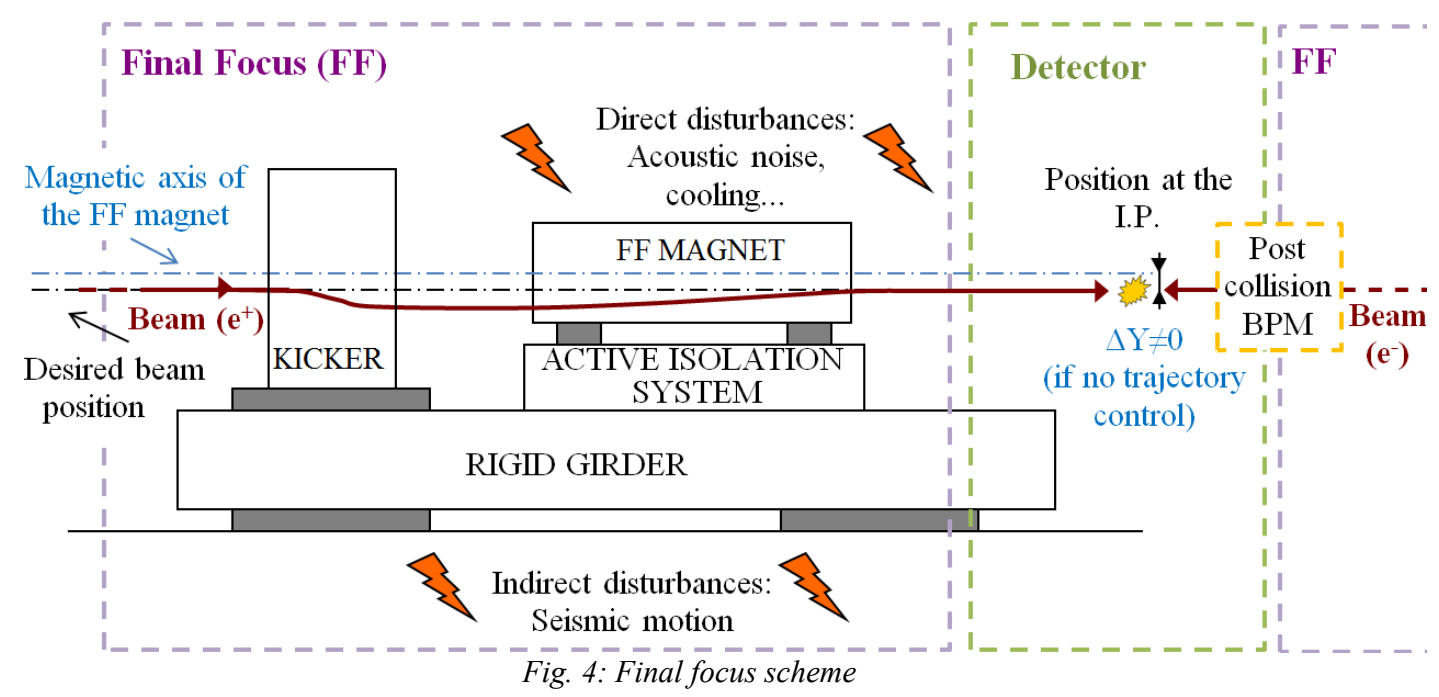

The kicker situated upstream from the IP on the incoming beam-line provides a corrective kick. As long as the field inside the kicker magnet is uniform over all the ground displacement, the effect of the latter on the beam kick can be neglected. 
The tight tolerances on the vertical displacement of the beam-line require real-time beam trajectory control to balance performance deterioration due to disturbance variations, temporal variations of the structure or model inaccuracy. The adopted strategy is a trajectory control with a feedback loop and a real-time adaptive control feature based on the Generalized Least Squares (GLS) algorithm (Landau, 2006) which helps in estimating and canceling the disturbance. The combination of both controls is designed to minimize the integrated RMS displacement of the beam. This strategy provides high damping, fast vibration suppression, good robustness and easy realization.

\section{Beam-based feedback}

The proposed control framework helps in designing an optimal controller minimizing the integrated RMS displacement of the beam. This optimization has to be done once, at the beginning, before the beam control starts. Numerical simulations on the whole system demonstrate how the proposed control minimizes vibrations caused by ground motion.

\subsection{Developed method}

Considering the process represented in figure 5:

- the backward shift operator is denoted $q^{-1}$,

- the disturbance $X$ is the mechanical excitation from ground motion on the FF magnet,

- the other direct disturbances $D$ on the FF magnet are added to the disturbance $X$,

- $P$ is the total disturbance felt by the FF magnet,

- the transfer function between the mechanical displacement of this magnet and the beam can be modeled by a constant matrix (which equals 1 in the model, assuming that the magnet is rigid enough),

- the BPM sensor noise $W$ is added to the beam displacement,

- the action $K_{b}$ meant to reduce the beam motion of the beam (or the offset between both beams at the interaction point) is done by a kicker $G$ which is located next to the magnets,

- the obtained beam displacement is proportional to the kicker-injected current (the proportionality equals 1 in the following model),

- the system dynamics is due to the frequency of the beam train, so the process $G$ can first be treated as a delay at a sampling period $T_{e}$ equal to $0.02 \mathrm{~s}$, 
- $H$ is the controller.

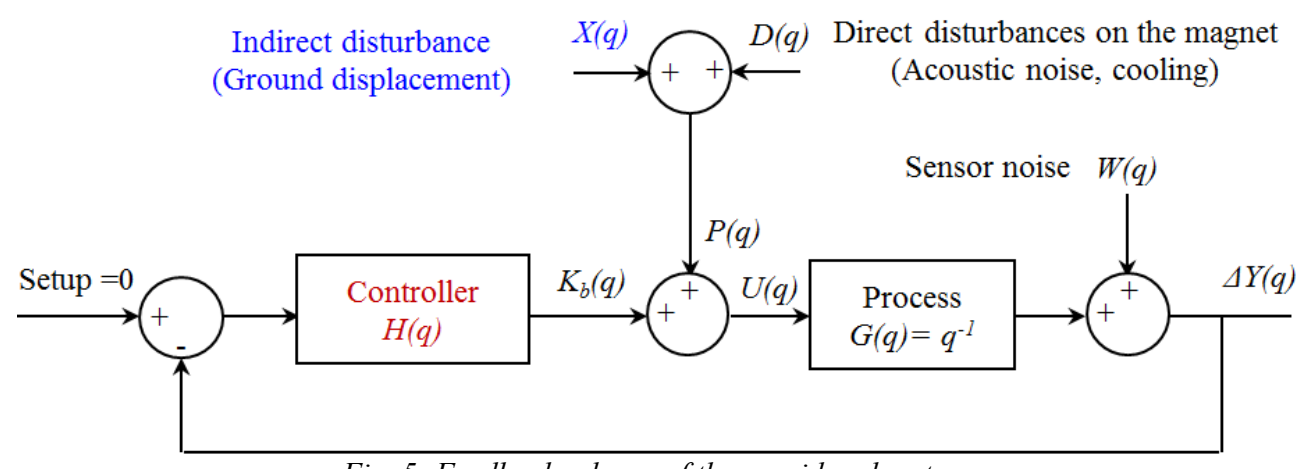

Fig. 5: Feedback scheme of the considered system

Considering the previous scheme, the closed loop transfer function taken into account is the transfer function $F$ (also called input sensitivity function) between the beam displacement and the disturbance $X$ :

$$
F=\frac{\Delta Y}{X}=\frac{\mathrm{G}}{1+\mathrm{G} H} .
$$

Note that as $G$ is a pure delay, the effect, in terms of amplitude of the closed loop on the sensor noise disturbance $W$, is the same as for the ground motion disturbance $P$.

\subsection{Optimization with linear controller}

The required specifications impose to lower the integrated RMS $(0.1 \mathrm{~Hz})$ (integrated RMS at $0.1 \mathrm{~Hz}$ ) using the controller. In order to tune the linear controller parameters, classical methods use pole placement or the frequency approach to obtain the performances in closed loop. In the case of CLIC, frequency considerations seem to be the right method. A controller based on the minimization of an $H^{\infty}$ performance norm (Doyle, 1989), (Bamieh, 1992) was initially designed but it did not perform well in practice. As a result, a simple controller structure was adopted:

$$
H(q)=\frac{b_{0}+b_{1} q^{-1}+b_{2} q^{-2}}{1+a_{1} q^{-1}+a_{2} q^{-2}}
$$

Higher order structures have been tested for the controller but they did not result in a significant gain in terms of integrated RMS, compared to the complexity of the feedback control. Thus a second order controller was chosen. 


\subsection{Tuning the controller parameters}

The method for lowering the integrated $\mathrm{RMS}(0.1 \mathrm{~Hz})$ of $\Delta Y$ runs through the following steps:

- estimation of the PSD for the measured ground motion signal,

- scanning the controller parameter space,

- for each of these combinations, if the parameters give a stable closed loop transfer function $F$, then:

- PSD computation of the obtained output using:

$$
\operatorname{PSD}(\Delta Y(\mathrm{j} \omega))=|F(\mathrm{j} \omega)|^{2} \operatorname{PSD}(X(\mathrm{j} \omega))
$$

- integrated RMS $(0.1 \mathrm{~Hz})$ computation,

- selection of the controller parameter set that gives the minimum integrated $\mathrm{RMS}(0.1 \mathrm{~Hz})$.

As these parameters obviously depend on the input sensitivity function $F$ and on the PSD of the input disturbance, if the input disturbance $X$ changes, then the optimization will produce another set of parameters. Note that in case of a non linear control (see part 4.1), the PSD of $\Delta Y$ is obtained by calculating the PSD of the output signal $\Delta Y$ (instead of using Eq. 4). However, this method is much more time-consuming in the proposed optimization case. In order to reduce the computation time, a computer cluster with 150 processors has been used. This approach does not necessarily lead to a global optimum, but the obtained integrated $\operatorname{RMS}(0.1 \mathrm{~Hz})$ is lower than the one obtained with the $H^{\infty}$ approach initially tested.

\subsection{Results}

The presented simulations have been computed without any other disturbance consideration than the ground motion disturbance signal $X$ taken from CMS data (Fig. 3). Given this configuration, the optimized parameter set is the following:

$$
a_{1}=-2 ; a_{2}=1 ; b_{0}=1.20 ; b_{1}=-1.28 ; b_{2}=0.24 \text {. }
$$

Note that the same technique has been tried on more complex structures (i.e. $3^{\text {rd }}$ order controller) but the integrated RMS varied marginally. Thus the previous controller structure was kept. 


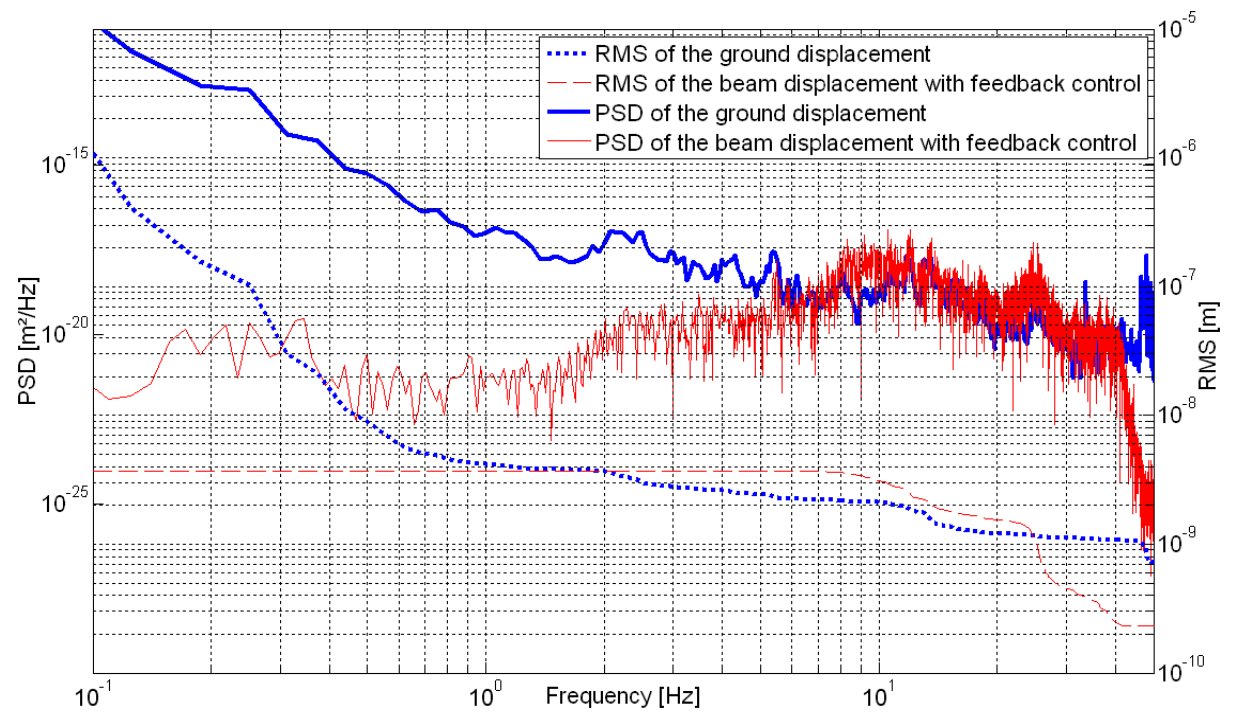

Fig. 6: Estimation of the PSD and integrated RMS displacement of the beam with a real disturbance and feedback control

Figure 6 represents the PSD and the integrated RMS displacements simulated with the optimized feedback control loop. It shows that the optimization technique has allowed to decrease the integrated $\mathrm{RMS}(0.1 \mathrm{~Hz})$ by a factor of 350 . High frequency displacements have been slightly amplified while low frequency displacements have been strongly reduced. This observation can be understood by looking at the transfer function $F$ between the ground motion disturbance and the output (see figure 7).

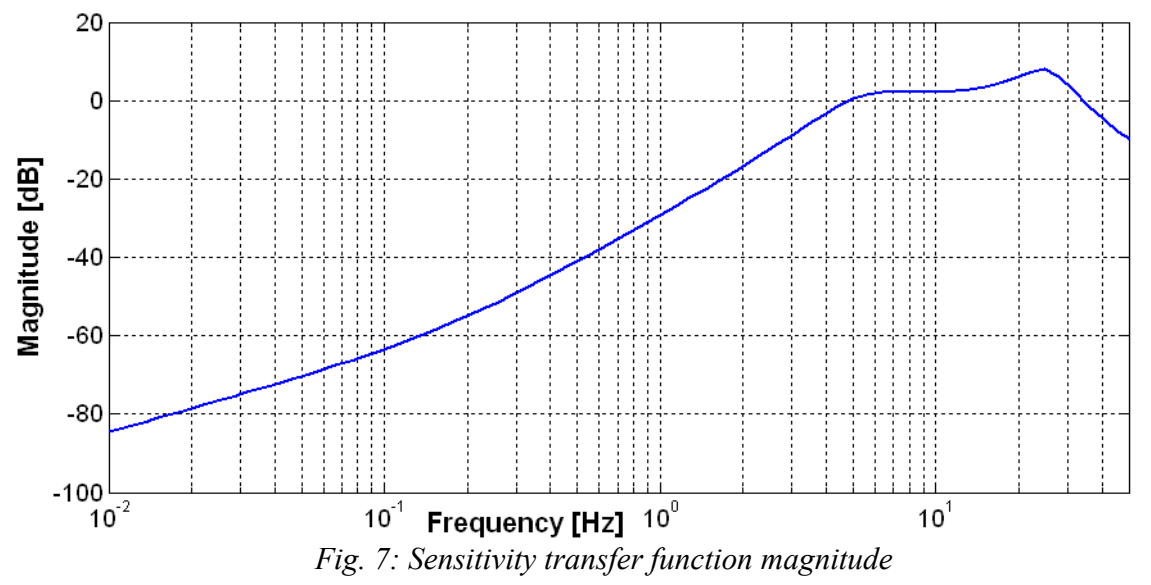

The transfer function $F$ must, and does, obey Bode's sensitivity relation (Mohtadi, 1990), that the integral of $\ln (F)$ is zero, meaning that reducing disturbances at low frequencies implies greater amplification of disturbances at high frequencies. The feedback efficiency is given by its ability to damp the ground motion vibrations in a given bandwidth. In this case, due to the sample time of the process, the feedback is efficient in a limited frequency bandwidth [0-5] Hz. It implies that the integrated RMS at $5 \mathrm{~Hz}$ of the disturbance $P$ has to be the lowest possible, and that a better value cannot be obtained with a Linear Time Invariant (LTI) controller for the proposed optimization. It is thus necessary to consider a complementary solution that could help in reducing the ground motion impact as much as possible. The results of figure 
6 show that the required specifications are not reached; $0.1 \mathrm{~nm}$ at $0.1 \mathrm{~Hz}$ is expected while about $4 \mathrm{~nm}$ is achieved (40 times higher).

Thus, in order to lower the integrated $\operatorname{RMS}(0.1 \mathrm{~Hz})$ of the output, three strategies are considered:

1) adding a measurement of the magnet position to implement a feed-forward or a feedback control,

2) minimizing the input disturbance $P$ by adding a mechanical filter,

3) integrating an adaptive controller with an estimation of the input disturbance in order to reject it.

In the frequency range $[1 \mathrm{~Hz}-1 \mathrm{kHz}]$, the first strategy may reduce the disturbance effect. But currently there are no valid solutions for such a measurement, due to the sensor precision needed, that is around $0.1 \mathrm{~nm}$, and due to the limited bandwidth of available sensors. However the beam deflection measurement used in this study can be precise enough with a realistic signal to noise ratio, but at the low rate of $50 \mathrm{~Hz}$. Note that this approach is not completely rejected by the physicists and that some studies are currently carried out in order to measure the magnet position. Nevertheless, it is not possible to obtain the steady-state position of the magnet with such sensors and this solution does not reject other disturbances (for example the bad position of the beam entering the FF), so a direct measurement of the beam-beam offset will still be necessary.

The second strategy is then needed if no other efficient loop can be added to lower the disturbance effect where the present control loop is not efficient. This can be done by adding an active/passive system supporting the magnets. In order to reduce the relative motion between the various magnets, two approaches have been carried out. The first one was to consider a single rigid structure supporting both the FF magnets, located on each side of the IP. If the ground motion is consistent enough, the two last focusing magnets would move in phase (Bolzon, 2009). However, the associated magnet vibration tolerances are severe (Assmann, 2000b) and unprecedented: the vertical integrated RMS displacement of the CLIC beam has to be lower or equal to 0.1 nanometer at $0.1 \mathrm{~Hz}$ and the distance between the last magnets on each side of the detector is large (about $12 \mathrm{~m}$ ). The rigid support approach has to be replaced by another scheme. As a consequence, the second approach will certainly be selected. It aims at integrating an isolation system in the transmittance between ground and the magnet, to reduce the motion of the magnet. It is quite simple to reduce the seismic noise effects above 5 $\mathrm{Hz}$ with a passive support but under this frequency, a support with a very large mass is needed. Current studies show that, under the constraint of the detector environment, a passive filter can be used with a cutoff frequency of around $1 \mathrm{~Hz}$. Active filters are also being studied but, in that case, it is difficult to obtain a good rejection of the disturbance above 50 
Hz. An isolator, currently being studied at CERN (Gaddi, 2010), will be used.

The third strategy may be a good solution because the disturbance is much more important at low frequency than at high frequency. The adaptive structure has the property to whiten the noise effect on the output. In order to obtain the same results with a LTI controller, a more complex controller with at least 3 integrators would be needed. But this would have some drawbacks: sensitivity to neglected dynamics and sensitivity to the variations of the dynamics of the mechanical support. The frequency response of the noise is quite flat around this frequency. When amplified by the control loop, it will lead to a bad integrated RMS $(0.1 \mathrm{~Hz})$, already too high at $10 \mathrm{~Hz}$. This kind of structure was unsuccessfully tried with this optimization technique or with the $H^{\infty}$ approach.

\section{Feedback and adaptive algorithm study}

\subsection{Adaptive algorithm}

Adaptive feedback is an attractive strategy when the disturbance sources are unknown or variable in time, which is the case in this study. Similar techniques have already been used in the accelerator field (Blanco, 2009). The approach followed here is to use an adaptive approach using a prediction-error to reconstruct and cancel out the disturbance $P$. The control scheme is given in figure 8 .

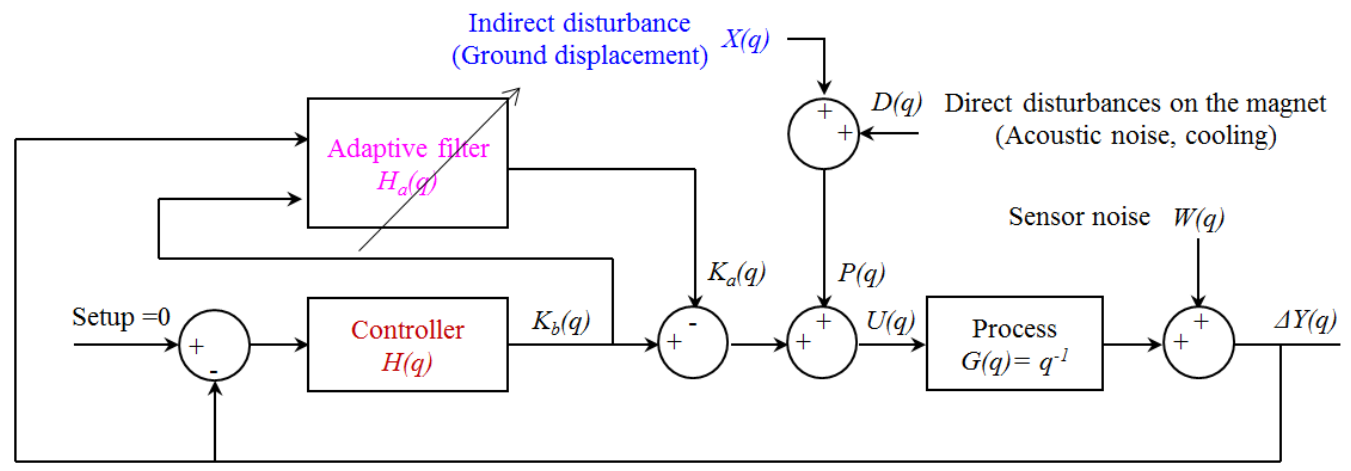

Fig.8: Feedback and adaptive control scheme

This scheme is non-linear as it is composed of two interlinked loops. A general adaptive command structure defined by Landau's (Landau, 2006) stability theorem has been used.

Using the fact that the process is a simple delay and neglecting the sensor noise, the prediction error to minimize at instant $n$ is then:

$$
e(n)=\Delta Y(n)-K_{b}(n-1)+K_{a}(n-1)-\boldsymbol{\phi}^{\mathbf{t}}(n) \boldsymbol{h}(n)
$$


where $\boldsymbol{\phi}^{\mathbf{t}}(n)$ is the vector of input/output signals of filter $H_{a}$ and $\boldsymbol{h}(n)$ its vector of parameters. The filtered error is given by:

$$
v(n)=\frac{e(n)}{1+\boldsymbol{\phi}^{\mathrm{t}}(n) \boldsymbol{F}(n) \boldsymbol{\phi}(n)},
$$

where $\boldsymbol{F}(n)$ is the adaptive gain given by:

$$
\boldsymbol{F}(n+1)=\frac{1}{\lambda_{1}}\left[\boldsymbol{F}(n)-\frac{\boldsymbol{F}(n) \boldsymbol{\phi}(n) \boldsymbol{\phi}^{\mathbf{t}}(n) \boldsymbol{F}(n)}{\frac{\lambda_{1}}{\lambda_{2}}+\boldsymbol{\phi}^{\mathbf{t}}(n) \boldsymbol{F}(n) \boldsymbol{\phi}(n)}\right] .
$$

Parameters $\lambda_{1}$ and $\lambda_{2}$ control the evolution of the adaptive gain. The new set of parameters is then given by:

$$
\boldsymbol{h}(n+1)=\boldsymbol{h}(n)+\boldsymbol{F}(n) \boldsymbol{\phi}(n) v(n)
$$

To obtain satisfactory results, re-optimization of the controller detailed in part 3.2 (including the adaptive algorithm) was necessary. This is due to the fact that the adaptive control and the feedback loop are both efficient in different bandwidths. It is then possible to optimize the global efficiency by adjusting these 2 bandwidths.

\subsection{Simulation results}

The optimization leads to the following parameters.

Parameters of the controller $H: \quad a_{1}=-2 ; a_{2}=1 ; b_{0}=-0.32 ; b_{1}=0.84 ; b_{2}=-0.48$.

Parameters of the adaptive control:

- forgetting factor $\lambda_{1}=0.99$ and $\lambda_{2}=1$,

- initial gain $\boldsymbol{F}(0)=10^{-6} \mathbf{I}$,

- control of the gain $\boldsymbol{F}: \max (\operatorname{trace}(\boldsymbol{F}))=10^{-4}, \min (\operatorname{trace}(\boldsymbol{F}))=10^{-6}$,

- the dimension of $\boldsymbol{h}$ is 1 .

Figure 9 gives the variation of the estimated parameter $\boldsymbol{h}$. Higher order estimated parameters have been tested but without better results. $\boldsymbol{h}$ varies around -1 in steady-state, but fixing $\boldsymbol{h}$ to -1 does not give satisfactory results. A delay, lower than 
the sampling period, was added in order to evaluate the computation time, and the simulations show identical performances.

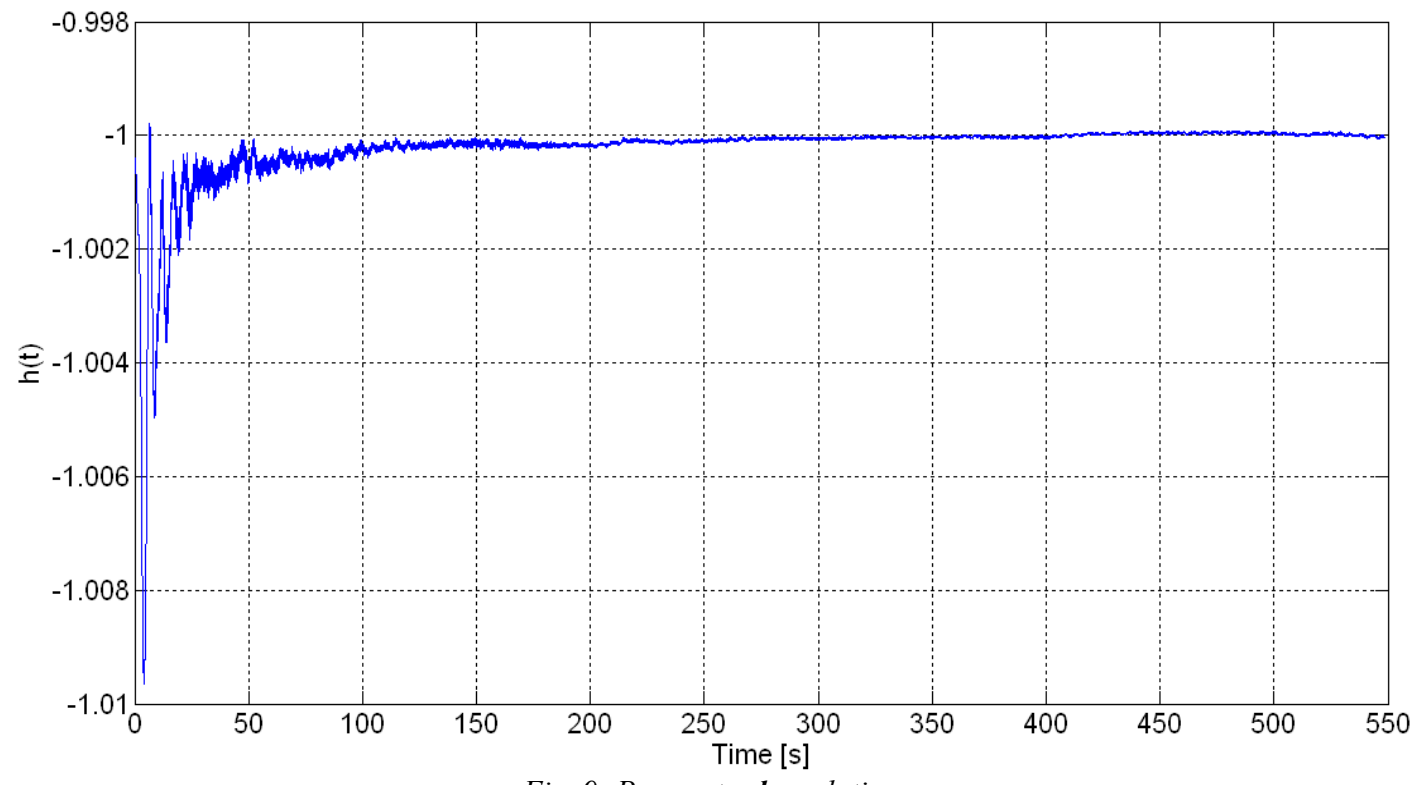

Fig. 9: Parameter $\boldsymbol{h}$ evolution.

Figure 10 represents the PSD obtained in simulation with and without adaptive control. The method combining feedback and adaptive control can considerably decrease the PSD displacement at low frequencies. Figure 11 gives the corresponding integrated RMS displacement.

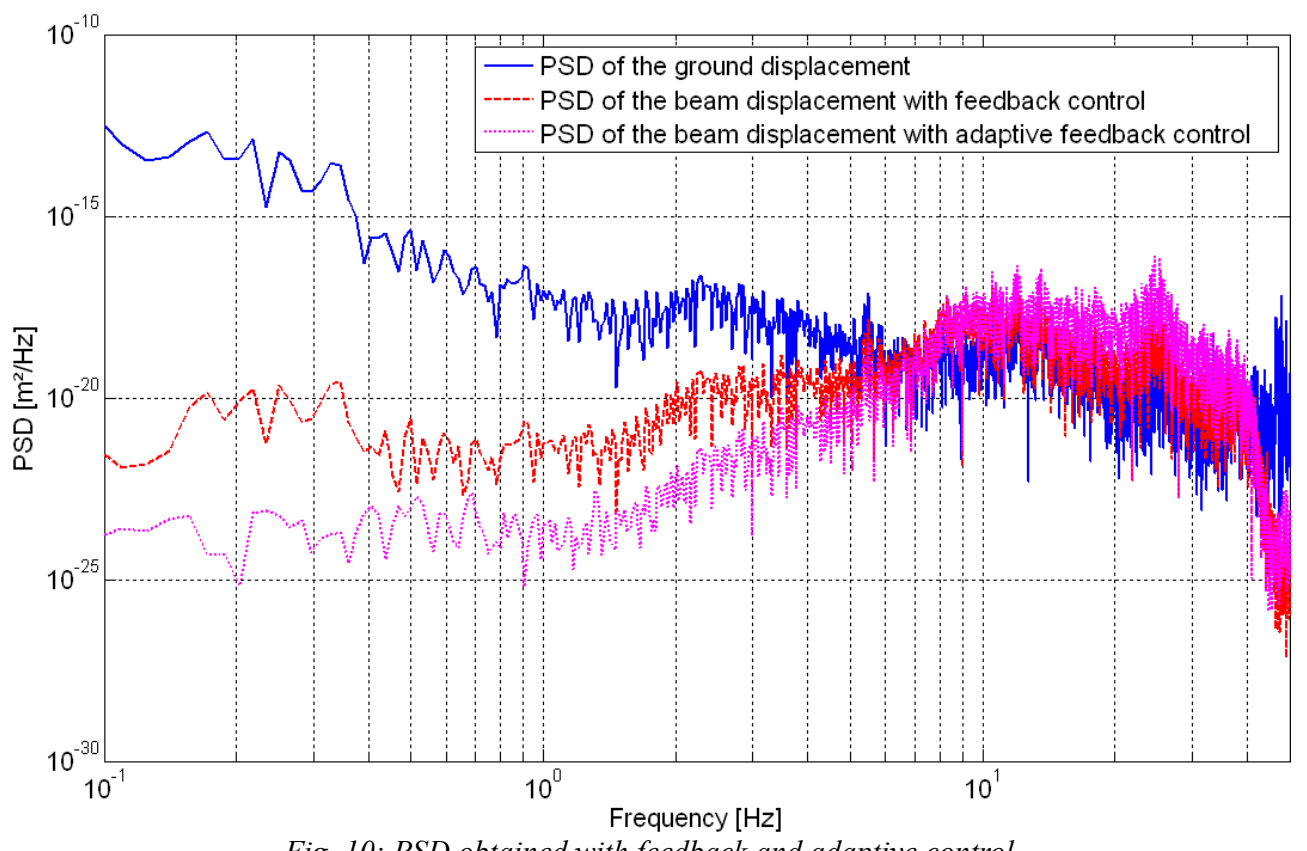

Fig. 10: PSD obtained with feedback and adaptive control

Although better performances can be achieved for low frequencies, the adaptive filter, albeit non linear, also obeys the Bode constraint, which causes an amplification after several Hertz. In the given configuration, this amplification has a detrimental effect on the integrated RMS, that reaches about $8 \mathrm{~nm}$. 


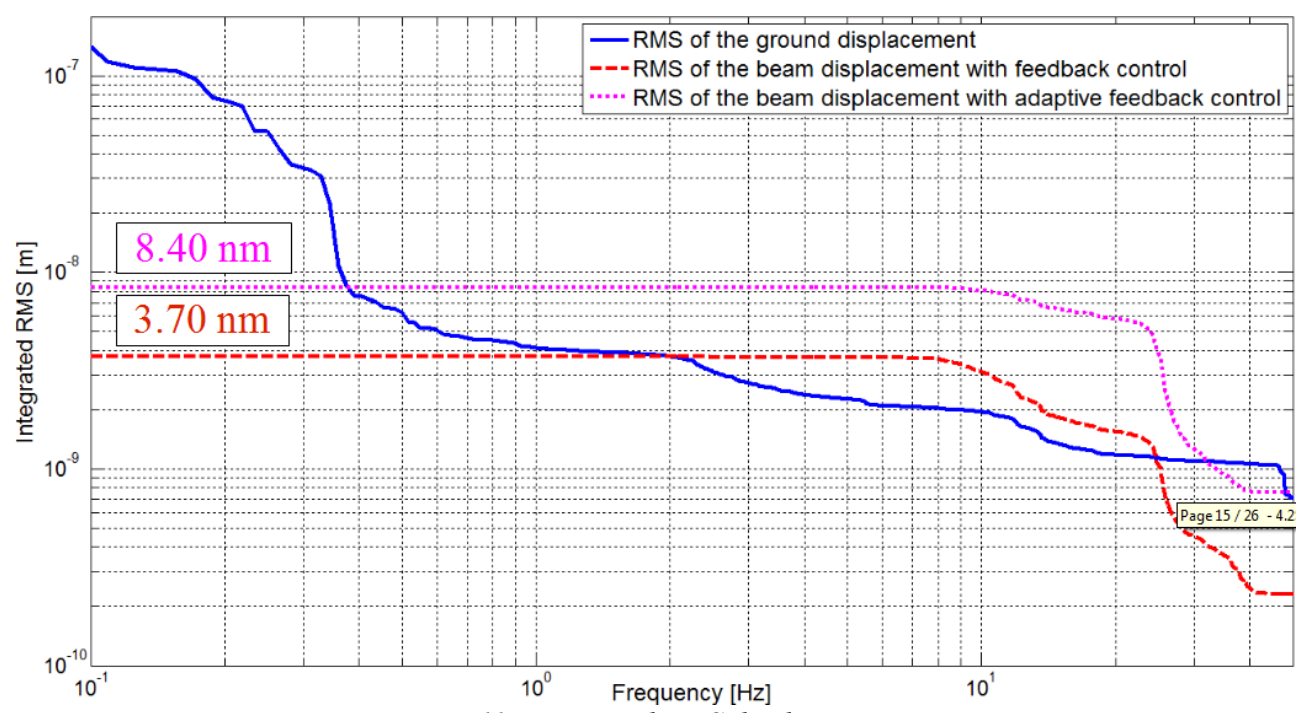

Fig. 11: Integrated RMS displacement

Previous observations with an optimized (adaptive) feedback loop show that the residual integrated $\mathrm{RMS}(0.1 \mathrm{~Hz})$ of the beam trajectory is mainly due to the integrated RMS at $5 \mathrm{~Hz}$ of the magnet support motion. Above that frequency, the feedback is not efficient. The strategy consists in adding an active/passive isolation $K$ (see figure 12) to simulate the presence of a flexible mechanical support under the magnets. It has to limit the ground motion vibration above $5 \mathrm{~Hz}$, which means that the resonance frequency of this mechanical structure has to be below $5 \mathrm{~Hz}$. The next part aims at creating a pattern of the transfer function $K$ needed between ground and the beam to reach the specifications. This transfer function should be representative of the dynamical behavior of a typical mechanical support. Such a pattern could be used for a more detailed study of a mechanical support design.

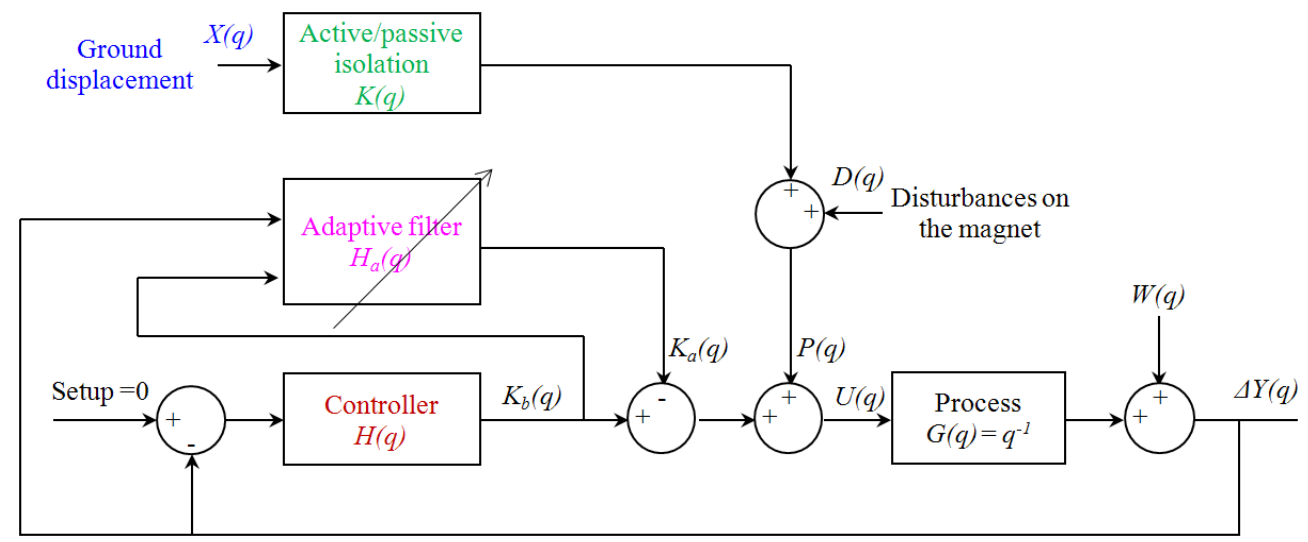

Fig. 12: Feedback and adaptive control scheme with active/passive damping 


\subsection{Pattern of the mechanical support}

In the following part the mechanical support $K$ has been assumed to be modeled by a $2^{\text {nd }}$ order low-pass filter:

$$
K(s)=\frac{G_{0}}{1+\frac{2 \xi}{\omega_{0}} s+\frac{1}{\omega_{0}^{2}} s^{2}} \text { with } \omega_{0}=2 \pi f_{0} .
$$

The strategy was to simulate the previous adaptive control algorithm when the ground motion disturbance is filtered by the mechanical filter $K$. By considering as a first approximation $\xi=0.7$, the aim is to find the combinations $\left(f_{0}, G_{0}\right)$ that can generate an integrated $\operatorname{RMS}(0.1 \mathrm{~Hz})<0.1 \mathrm{~nm}$. As the input sensitivity function now depends on $K$, it was necessary for each combination to optimize the controller until the specifications are reached (see part 3.3). Figure 13 shows all these combinations.

The area under the curve represents the possible combinations of $\left(f_{0}, G_{0}\right)$. Due to complex and long computations, this curve is probably lightly under-estimated (the controller is not fully optimized). The number of combinations being slightly greater, the difference can be evaluated to about $5 \%$. Another approach was to study the influence of the damping ratio $\xi$ on the integrated $\operatorname{RMS}(0.1 \mathrm{~Hz})$ instead of considering it equal to 0.7 . Calculations show that the previous curve is quite independent from the variations of $\xi$ in the range [0.01- 0.7]. This comes from the extremely high efficiency of the adaptive algorithm at low frequency. This passive support, if feasible, could be efficient enough to achieve the desired performances.

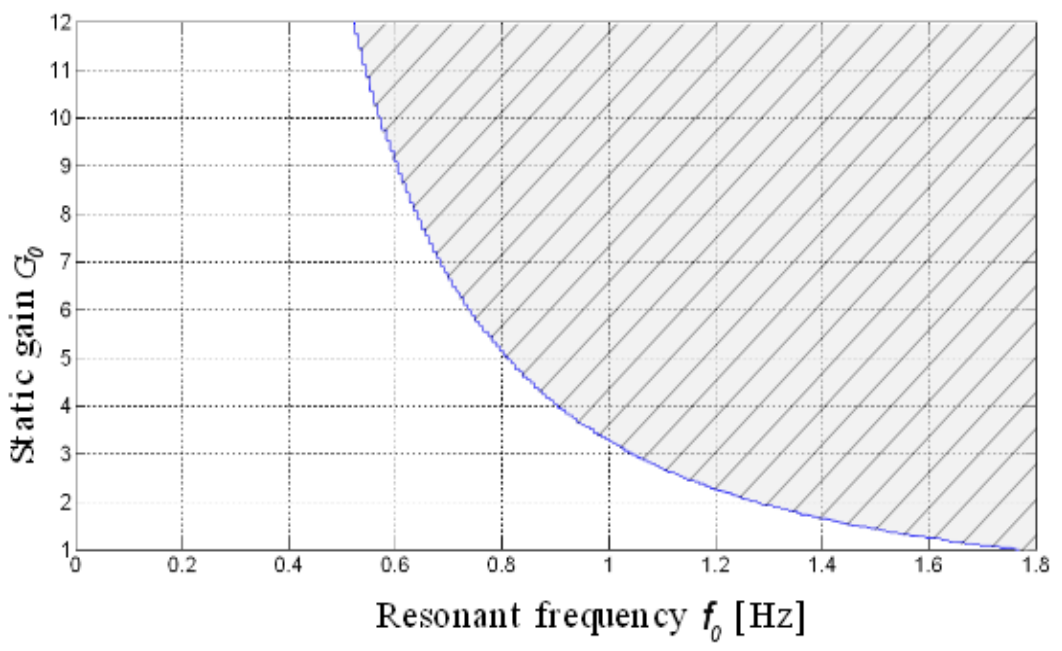

Fig. 13: Pattern of the mechanical support behavior 


\subsection{Mechanical support considerations}

Different solutions combining active and passive isolation already exist. For example, this strategy has been widely used in industrial fields (Schulz, 2003) such as microscopy, optics, aeronautics. A lot of solutions have been studied in particle physics (Seryi, 2001) as well. A study (Redaelli, 2003) shows that one of the most efficient solutions on the market for this type of application is the TMC table ${ }^{4}$ with STACIS feet. It integrates rubber dedicated to the passive isolation in order to filter the vibrations, and an active system composed of piezoelectric-actuators and geophones used within a feedback circuit. This product can manage vibrations at a sub-nanometer scale, and has also been tested in simulation. Its transfer function:

$$
K(q)=\frac{0.2664-0.7629 q^{-1}+0.7282 q^{-2}-0.2317 q^{-3}}{1-2.212 q^{-1}+1.427 q^{-2}-0.2149 q^{-3}}
$$

has been obtained by identification. Figure 14 represents the PSD and the integrated RMS displacement obtained by simulation of the active isolation.

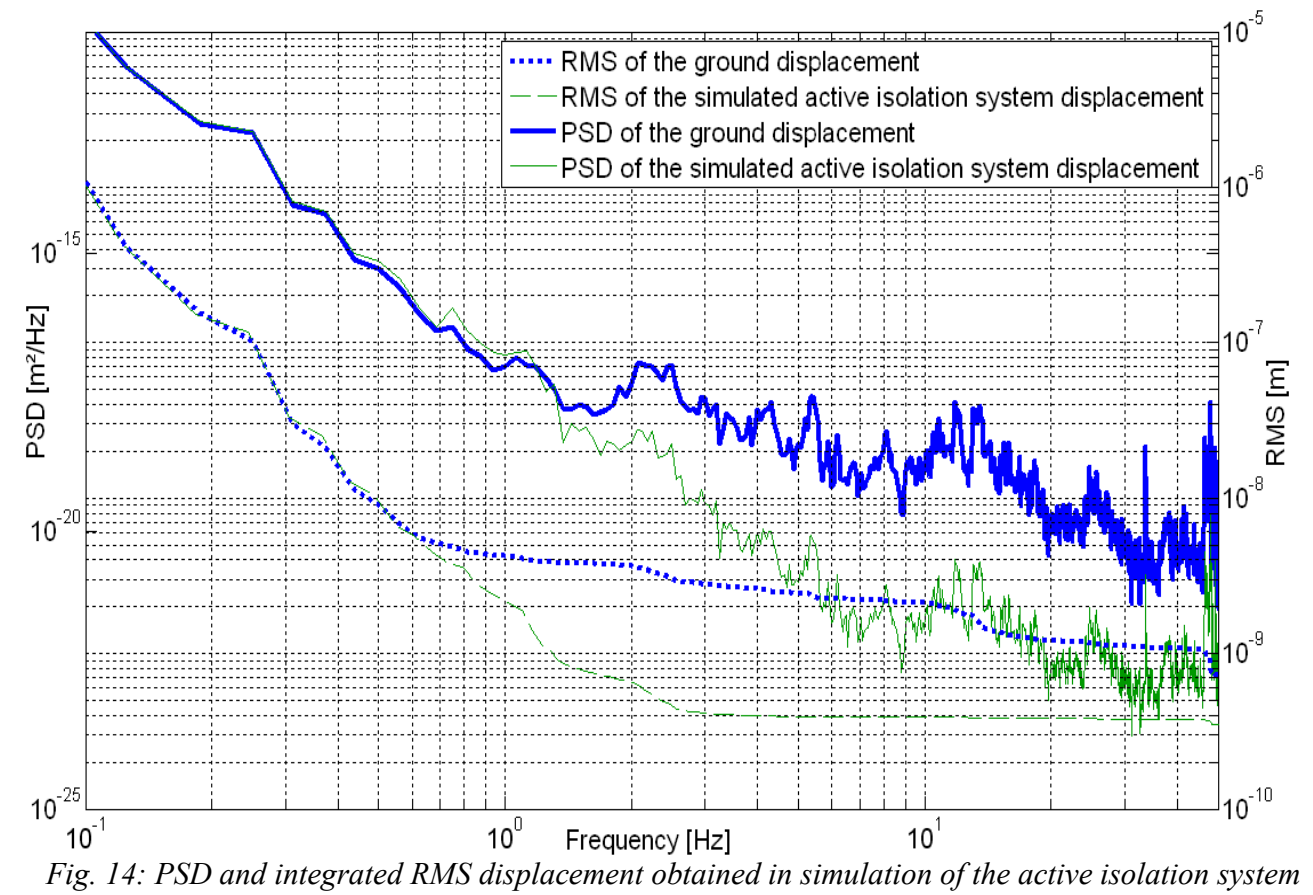

Damping was achieved in the frequency range 1.3-50 Hz. However, the TMC table is useless for this application under $1.3 \mathrm{~Hz}$ because it amplifies ground vibrations. The model of the active isolation system enables to decrease the disturbances' integrated RMS $(5 \mathrm{~Hz})$ to about $0.4 \mathrm{~nm}$. This is already too much according to the specification of $0.1 \mathrm{~nm}$ at $0.1 \mathrm{~Hz}$. In addition, the integrated $\mathrm{RMS}(0,1 \mathrm{~Hz})$ remains the same with or without active isolation. This is due to the fact that the main contribution to the integrated $\operatorname{RMS}(0,1 \mathrm{~Hz})$ comes from the high PSD amplitudes of ground motion at low frequencies below $2 \mathrm{~Hz}$ following a $1 / f^{4}$ behaviour. 
Given the tight specifications, a dedicated support has to be designed. The design of such a mechanical filter is not dealt with in this study but some concepts have already been developed (Braccini, 2005). In the simulated layout, the final focus magnet has been considered to be placed on a passive system whose behavior is similar to that of the mechanical filter described in 4.3. This support has been incorporated in the following simulations. In order to fit the pattern in figure 13, the parameter values $\omega_{0}=2 \pi \mathrm{rad} / \mathrm{s}$ and $G_{0}=1$ have been used and the worst case $\xi=0.01$ has been chosen. Figures 15 and 16 represent the simulation results of the feedback control obtained by adding this mechanical support.

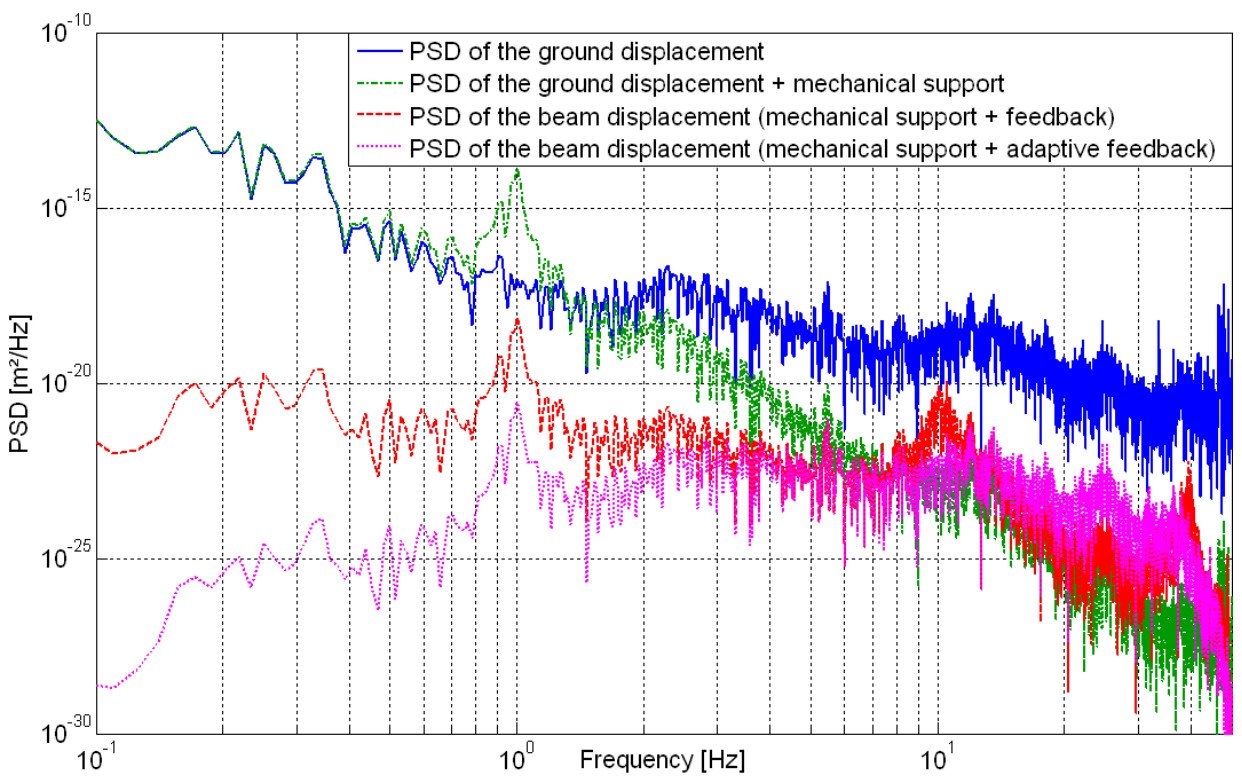

Fig. 15: PSD obtained with mechanical support, feedback and adaptive control

This strategy, combining feedback and adaptive control, can considerably decrease the PSD displacement for low frequencies. Results show that it can promisingly be associated with the previous mechanical support, as it brings a damping of the beam motion on a larger frequency bandwidth. Figure 16 provides the integrated RMS displacement.

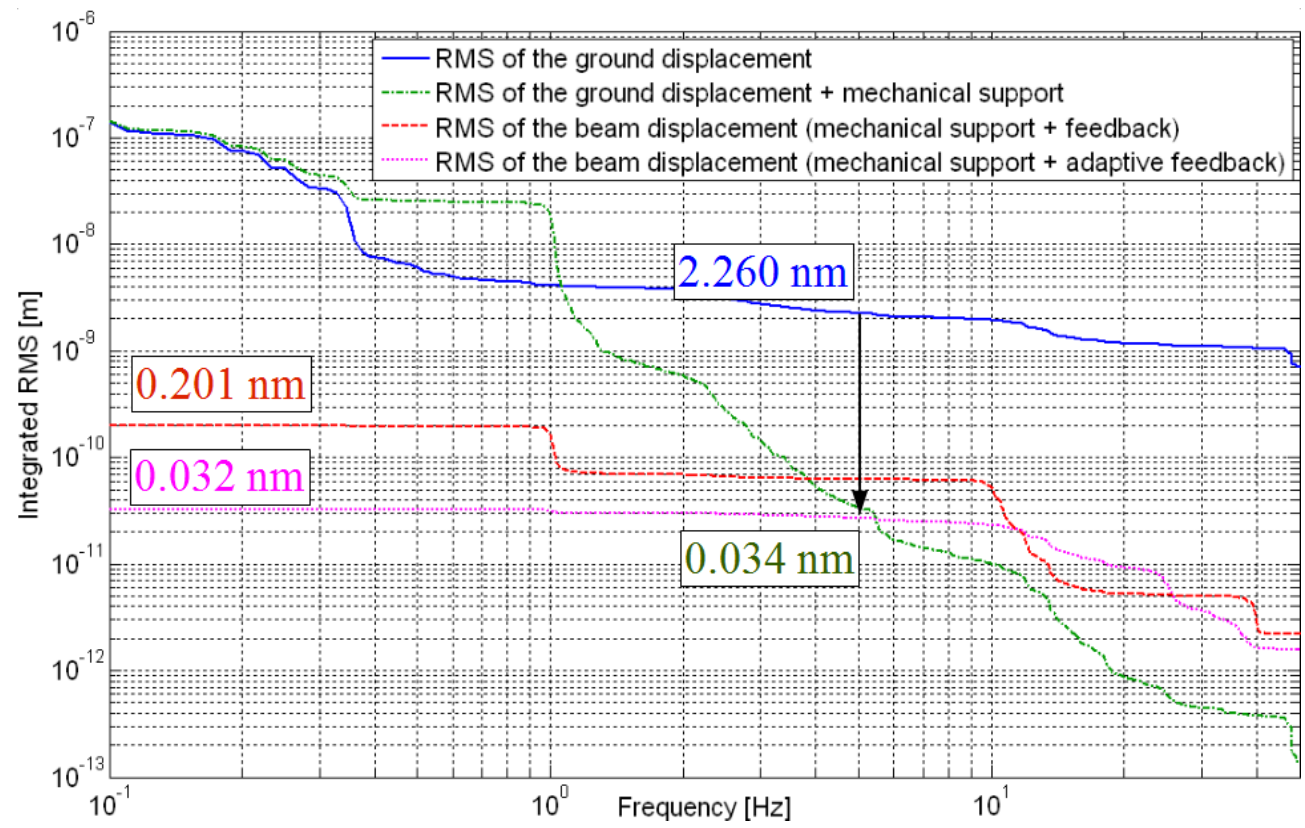

Fig. 16: Integrated RMS obtained with mechanical support, feedback and adaptive control 
The integrated RMS(5 Hz) of the ground motion indeed dropped from $2.26 \mathrm{~nm}$ to $0.03 \mathrm{~nm}$ with the mechanical support. Moreover, the integrated $\mathrm{RMS}(0.1 \mathrm{~Hz})$ of the beam displacement also dropped from $3.70 \mathrm{~nm}$ to $0.20 \mathrm{~nm}$ with the feedback loop and from $8.40 \mathrm{~nm}$ to $0.03 \mathrm{~nm}$ with the adaptive feedback loop (which means respectively a factor 20 and 200). This result proves that the adopted strategy which consists in using a passive isolation to damp fast motion of the ground, and a feedback loop coupled with an adaptive algorithm dealing with slower motions is a very efficient solution to meet the requirements.

\subsection{Robustness}

The previous study gives a model of the real system. Considering that the project is in its conceptual design phase, it was necessary to make several hypotheses and to arbitrarily set certain parameters. A first study aims at comparing the integrated RMS displacement obtained if the mechanical support $K$ is not properly characterized (model inaccuracy, parameter drifts). As it is considered as a resonant second-order low-pass filter, two parameters must be taken into account; the damping factor $\xi$ and the resonant frequency $f_{0}$, both depending on the proper characteristics of the support. Figure 17 shows the evolution of the integrated RMS displacement for a variation of these parameters when the controller has been optimized for the mechanical support $\left(f_{0}=1 \mathrm{~Hz}, \xi=0.01, G_{0}=1\right.$ and integrated $\left.\operatorname{RMS}(0.1 \mathrm{~Hz})=0.04 \mathrm{~nm}\right)$.

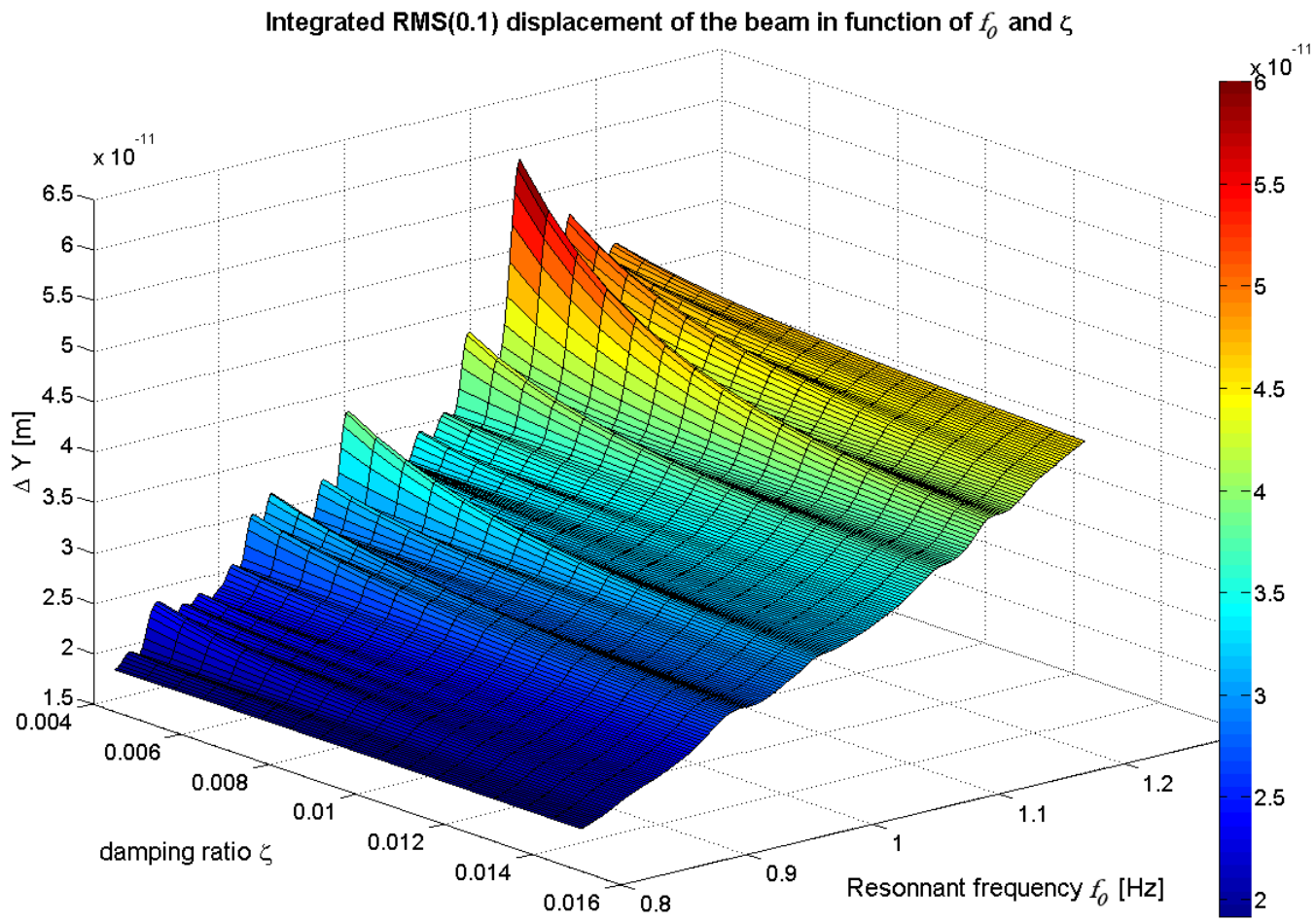

Fig. 17: Integrated RMS $(0.1 \mathrm{~Hz})$ versus mechanical support characteristics

The simulation has been computed for a variation of $\pm 10 \%$ and $\pm 50 \%$ for $f_{0}$ and $\xi$. Several "waves" representative of the various excitation modes of the process can be observed. The worst case implies an integrated RMS $(0.1 \mathrm{~Hz})$ of about 0.06 
$\mathrm{nm}$, which is still under the requirements. These results show that the global control has a good robustness with respect to unmodelled or neglected dynamics.

The second robustness study was dedicated to the instrumentation used and more precisely to the BPMs which are still being developed. The aim is to estimate the performances needed for this monitor and mainly which level of internal noise will be acceptable without decreasing too much the efficiency of the control. Figure 18 shows the variation of the beam integrated RMS displacement as a function of the BPM noise (represented by a white noise added to the measured displacement).

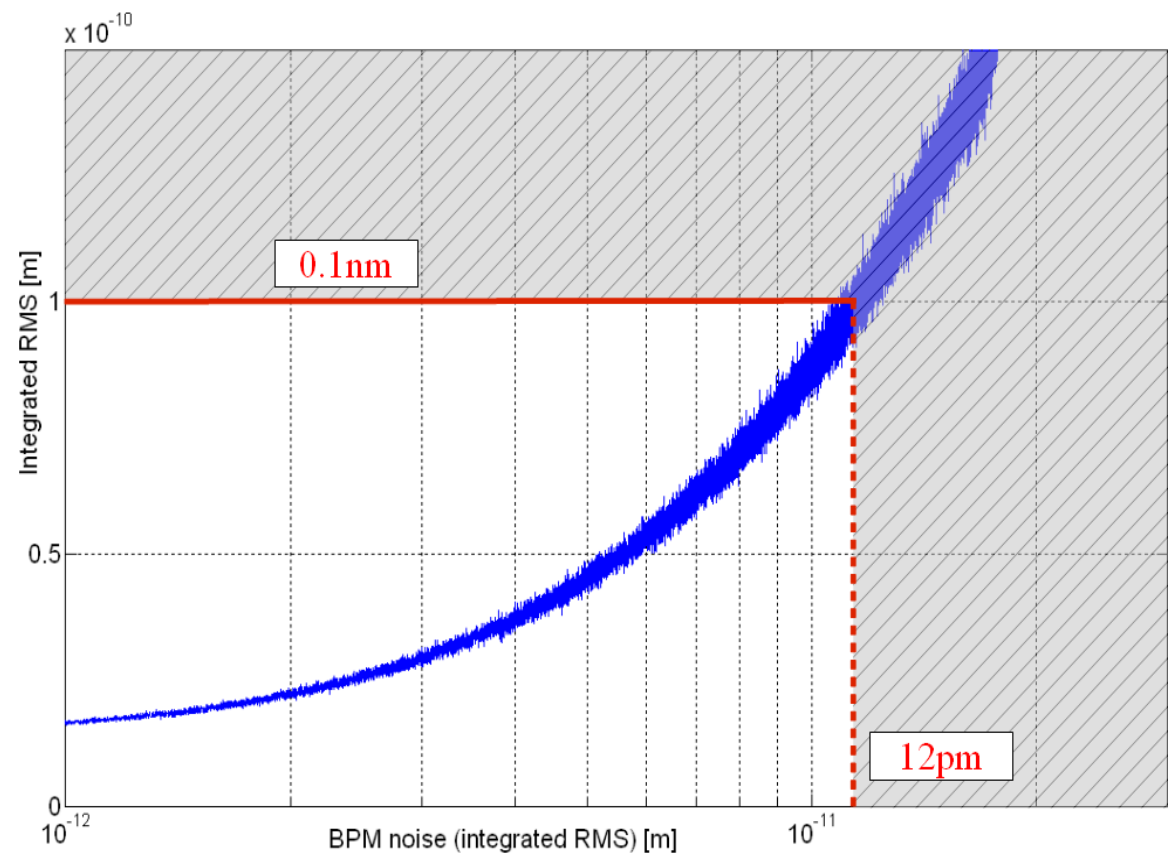

Fig. 18: Variation of the integrated RMS displacement at $0.1 \mathrm{~Hz}$ versus BPM noise

This study reveals that even a low monitor noise has detrimental effects on the control. In order to reach the specifications, the level of the sensor noise has to be lower than the equivalent of a 12 picometer displacement at the IP integrated RMS at $0.1 \mathrm{~Hz}$. As the BPM gives an indirect measurement (position image multiplied by $10^{5}$ because of its distance from IP), the BPM noise has to be $<1.2 \mu \mathrm{m}$ in order to preserve the integrated $\mathrm{RMS}(0.1 \mathrm{~Hz})$. This result has to be taken into consideration in the design of this monitor and will certainly be one of the strategic requirements.

\subsection{Further development}

All the presented results are based on simulation tests, as this is usually the case in this kind of development where the time to design large instruments spans several tens of years. The next stage consists obviously in carrying out a representative mock-up to demonstrate that all the assumptions are respected and to confirm the simulated results. Usually, in particle physics field, the demonstration is made in several stages. 
The first one is to transpose the developed simulations, which were done with standard tools, in a common simulation platform dedicated to a specific collider. It integrates all the collider subsystems and their associated actions to the beam physics, taking into account all the acquired experience with the colliders previously built, as the CERN's LHC (Virdee, 2010) for example. In the case of the CLIC experiment, this tool is named PLACET (Schulte, 2000) and will give the opportunity to test the interfaces between the various subsystems. The integration of the presented developments to this software is ongoing as the other subsystems will be tested as soon as possible.

In parallel, two dedicated linear "short" colliders were built to prove its feasibility. The first one is ATF2 (Accelerator Test Facilities) (Seryi, 2009) in Japan and was commissioned in 2008. However its current beam repetition rate of $1 \mathrm{~Hz}$ doesn't fit the needs of this study. The second one is CTF3 (CLIC Test Facility 3) (Geschonke, 2002) at CERN. It should be commissioned by 2012. In this context, the CLIC physics community studies the possibility to test certain subsystems on colliders but the unprecedented desired size of the beam generates some specific problems (Gasior, 2010) and none seems to be adapted to test the simulations of this study.

The last possibility is to test the adaptive feedback control on a laser beam or on an interferometric system but it will not be possible to test the interfaces with the other subsystems. Indeed, some recent PLACET simulations have shown that this control interferes with other feedbacks (which could generate high frequency ground motion amplification). Thus, the strategy of the physics community, is to upgrade the ATF2 or CTF3 but well beyond 2012.

\section{Conclusions}

The feasibility of the future linear collider CLIC has to be proved in different aspects: one of them consists in showing that the collision of beams in the sub-nanometer scale is feasible. Considering that the imposed tolerances (integrated $\operatorname{RMS}(0.1 \mathrm{~Hz})$ at the interaction point has to be lower than $0.1 \mathrm{~nm})$ are considerably lower than the natural ground motion (a few micrometers), these requirements are very challenging and were never achieved in the past. A mechanical setup and a dedicated control approach have been presented. They lead to a very low vertical beam displacement at the interaction point with an integrated $\mathrm{RMS}(0.1 \mathrm{~Hz})$ of about $0.04 \mathrm{~nm}$. To reach these results, the strategy was to study an innovative control which is very efficient at low frequencies. This algorithm combines a feedback obtained after a parametric study and an adaptive control based on the generalized least-squares method. This method was tested in simulation with a representative model of the system and with real measurement of the ground motion. Then, a pattern of the dynamical behavior of the required mechanical damping structure needed to filter the vibrations above a few Hertz 
has been established to guide further development. To validate this study, simulation tests have been performed with a mechanical support, as well as robustness tests in order to take into account the prediction errors of the mechanical system model and to estimate the acceptable maximal sensor noise. These simulation results need to be tested experimentally. This will be done over the next few years on one of the CLIC Test Facilities meant to demonstrate the technical feasibility of the key concepts of the novel CLIC.

\section{Acknowledgments}

The research leading to these results has received funding from the European Commission under the FP7 Research Infrastructures project EuCARD, grant agreement no. 227579. The authors wish to thank D. Schulte, J. Pfingstner and K. Artoos from CERN, for this project and their fruitful collaboration. Grateful acknowledgment is made to G. Deleglise and L. Pacquet from LAPP for their helpful feedback and support.

\section{Nomenclature Tables}

ATF2

BPM

CLIC

CMS

CTF3

$\mathrm{FF}$

GLS

IP

LAPP

LHC

LTI

ML

PSD

RMS

$D$

F

G

H

$H_{a}$

K
Accelerator Test Facilities

Beam Position Monitor

Compact Linear Collider

Compact Muon Solenoid

CLIC Test Facility 3

Final Focus

Generalized Least-Square

Interaction Point

Laboratoire d'Annecy-Le-Vieux de Physique des Particules

Large Hadron Collider

Linear Time Invariant

Main Linac

Power Spectral Density

Root Mean Square

Direct disturbance on the magnet

Input Sensitivity Function

Kicker (actuator used to steer the beam)

Numerical controller

Adaptive filter

Mechanical filter (active-passive isolation) 


$\begin{array}{ll}K_{a} & \text { Corrective action provided by the adaptive filter } \\ K_{b} & \text { Corrective action provided by the controller } \\ P & \text { Disturbance felt by the magnet } \\ W & \text { Noise of the sensor } \\ X & \text { Mechanical excitation from ground motion } \\ \Delta Y & \text { Relative displacement between the beams } \\ \xi & \text { Damping ratio } \\ G_{0} & \text { Static gain } \\ f_{0} & \text { Resonant frequency } \\ \omega_{0} & \text { Resonant pulsation } \\ \mathrm{q}^{-1} & \text { Backward shift operator } \\ \mathrm{T}_{\mathrm{e}} & \text { Sampling period of the process }\end{array}$

\section{References}

Adloff, C. et al., 2005. Vibration stabilization for the final focus magnet of a future linear collider. 6th International Workshop on Research and Education in Mechatronic (REM'05), Annecy-le-Vieux, France.

Assmann, R. et al., 2000a. Stability Considerations for Final Focus Systems of Future Linear Colliders. Proceedings of European Particle Accelerator Conference (EPAC'00), Vienna, Austria.

Assmann, R.W. et al., 2000b. A 3 TeV e+ e- Linear Collider Based on CLIC Technology. CERN Document Server, CERN-2000-008.

Bambade, P. et al., 1989. Observation of beam-beam deflections at the interaction point of the SLAC linear collider. Physical review letters, vol. 62 Issue 25 pp.2949-2952.

Bamieh, B.A, Pearson, J.B., 1992. A General Framework for Linear Periodic Systems with Applications to Sampled-Data Control. IEEE Transactions on Automatic Control, Vol. AC-37, pp. 418-435.

Bialowons, W. et al, 2006. Measurement of ground motion in various sites. Proceedings of European Particle Accelerator Conference (EPAC'06), Edinburgh, UK.

Blanco, E. et al., 2009. Nonlinear predictive control in the LHC accelerator. Control Engineering Practice, Volume 17, Issue 10, Pages 1136-1147, ISSN 0967-0661.

Bolzon, B. et al., 2005. Modeling of simple cases in view of active stabilization for a future linear collider. Nanobeam 2005, Kyoto, Japan.

Bolzon, B., 2006. Etude des vibrations et de la stabilisation à l'échelle sous-nanométrique des doublets finaux d'un collisionneur linéaire. Ph. D., France, Université de Savoie, LAPP-T-2007-05.

Bolzon, B. et al., 2008. Study of vibrations and stabilization at the sub-nanometer scale for CLIC final doublets. Nanobeam 2008, Novosibirsk, Russia, Advanced Beam Dynamics Workshop: LAPP-TECH-2008-05.

Bolzon, B., 2009. Linear collider final doublet considerations: ATF2 vibration measurements. Proceedings of Particle Accelerator Conference (PAC'09), Vancouver, BC, Canada, TH5RFP087.

Braccini, S., et al., 2005. Measurement of the seismic attenuation performance of the VIRGO Superattenuator. Astroparticle Physics 23 , pp. 557-565.

Braun, H. et al. 2008. CLIC 2008 parameters. CERN Document Server, CLIC-Note-764.

Burrows, P.N. et al., 2007. Electromagnetic background tests for the ILC interaction-point feedback system. IEEE Proceedings of Particle Accelerator Conference (PAC'07), vol. 1-11 pp.3757-3759.

Dalena, B., Schulte, D., 2010. Beam-beam background in CLIC in presence of imperfections. Proceedings of International Particle Accelerator Conference (IPAC'10), Kyoto, Japan.

CMS Collaboration, 2008. The CMS experiment at the CERN LHC. Journal of Instrumentation, 3S08004 pp.26-89. 
Doyle, J.C, Glover, K., Khargonekar, P., Francis, B., 1989. State-space solutions to standard H2 and Hœ control problems. Transactions on Automatic Control vol. 34, no. 8, August, pp. 831-847

Gaddi, A., Gerwig, H., Siegrist, N., Ramos, F, 2010. Dynamic analysis of the FF magnets pre-isolator and support system. EDMS Document Server, EDMS n 1098581.

Gasior, M. et al., 2010. Sub-nm beam motion analysis using a standard BPM with high resolution electronics. Proceedings of BIW10, Santa Fe, New Mexico, USA, 2005.

Geschonke, G., 2003. CTF3 Design Report. CERN Document Server, SLAC-PUB-9672.

Goldstein, D., Saltzberg, D., 2003. The RASNIK real-time relative alignment monitor for the CDF inner tracking detectors. Nuclear Instruments and Methods in Physics Research A, vol. 506, pp. 92-100.

Landau, I.D., Zito, G., 2006. Digital Control Systems: Design, Identification and Implementation. Communications and Control Engineering, Springer Verlag.

Mohtadi, C., 1990. Bode's integral theorem for discrete-time systems. IEE proceedings. Part D. Control theory and applications, vol. 137, no2, pp. 57-66.

Redaelli, S., 2002. The effect of cooling water on magnet vibrations. Proceedings of European Particle Accelerator Conference $\left(E P A C^{\prime} 02\right)$, Paris, France.

Redaelli, S. et al., 2003. Colliding nanobeams in CLIC with magnets stabilized to the sub-nm levels. PAC 2003, CERN , Geneva, Switzerland.

Schulte, D., 2000. PLACET - A program to simulate drive beams. Proceedings of European Particle Accelerator Conference (EPAC'00), Vienna, Austria.

Schulte, D., Zimmermann, F., 2001. The Crossing Angle in CLIC. Proceedings of Particle Accelerator Conference (PAC'01), Chicago, USA.

Schulz, G., 1979. Active multivariable vibration isolation for a helicopter. Automatica, 15(4), 461-466.

Seryi, A., 2001. Ground motion and vibration issues for accelerators. IEEE Proceedings of Particle Accelerator Conference (PAC'01), Chicago, USA.

Seryi, A., et al., 2009. ATF2 Commissioning. Proceedings of Particle Accelerator Conference (PAC'09), Vancouver, Canada, FR1RAI03.

Sjöström, M., Wallén, E., Eriksson, M., Lindgren, L.-J., 2007. Corrector-based magnet alignment procedure. Nuclear Instruments and Methods in Physics Research A, vol. 597, pp.170-177.

Virdee, T.S., 2010. The LHC project: The accelerator and the experiments. Nuclear Instruments and Methods in Physics Research A, vol. 623, no1, pp. 1-10. 